\title{
Membership of the ICSB Subcommittee on Listeria
}

H. J. Welshimer, Chairman

Medical College of Virginia

Virginia Commonwealth University

MCV Station

Richmond, Virginia 23298, U.S.A.

Dorothy Jones

Department of Microbiology

Schools of Medicine and Biological Sciences

The University

Leicester LE1 7RH, United Kingdom

H. Seeliger

Institut für Hygiene und Mikrobiologie der

Universität Würzburg

Josef-Schneider-Strasse 2,

Bau 17, Würzburg, Federal Republic of Germany

I. Ivanov

Central Veterinary Research Institute

15, P. Slaveikov Blvrd.

1606 Sofia, Bulgaria

\section{A. L. Courtieu}

Universite de Nantes

Bacteriologie et Hygiene

1 Rue Gaston Veil

Nantes 44035, France

B. S. Ralovich

Institutum Sanitatis

Publicae et Epidemiologiae Universitatis

7643 - PECS

Szigeti ut 12 , Hungary

M. Woodbine

Department of Applied Biochemistry and $\mathrm{Nu}$ trition

$\dagger$ Members with especial interest in Erysipelothrix.
School of Agriculture

Sutton Bonington

Loughborough LE12 5RD

Leicestershire, United Kingdom

$\dagger$ E. W. Ewald

Paul-Ehrlich-Institut

6000 Frankfurt-am-Main 70

Paul-Ehrlich-Strasse 42-44, Federal Republic of Germany

† M. Truszczynski

Director of Veterinary Research Institute

Partyzantow 57

24-100 Pulawy, Poland

† G. Kucsera

State Institute for the Control of Veterinary Serobacteriological Products

H-1107 Budapest X.

Szallas UTCA 8, Hungary

R. G. Dijkstra

Animal Health of Friesland

Kruistraat 41

Leeuwarden, The Netherlands

S. Ortel

Direktor des Instituts für Medizinische Mikrobiologie und Epidemiologie

Martin-Luther Universität Halle-Wittenburg

402 Halle (Saale)

Leninallee 6, German Democratic Republic

I. E. Bakulov

All Union Research Institute of Veterinary, Virology and Microbiology

Pokrov 601120

Vladimirskaya oblast, U.S.S.R. 